\title{
Microscopical Characterization of Cast Hypereutectic Al-Si Alloys Reinforced with Graphene Nanosheets
}

\author{
A. S. Alghamdi \\ Mechanical Engineering Department \\ College of Engineering \\ University of Hail \\ Hail, Saudi Arabia \\ Asbg945@hotmail.com
}

K. S. Abdel Halim

Chemical Engineering Department, College of Engineering University of Hail, Hail, Saudi Arabia and

Central Metallurgical Research and Development Institute Cairo, Egypt

k.abdulhalem@uoh.edu.sa

\author{
Mohamed Ramadan \\ Mechanical Engineering Department, College of Engineering \\ University of Hail, Hail, Saudi Arabia and \\ Central Metallurgical Research and Development Institute \\ Cairo, Egypt \\ mrnais3@yahoo.com
}

Naglaa Fathy

Physics Department

College of Science

University of Hail

Hail, Saudi Arabia

Naglaaf2002@yahoo.com

\begin{abstract}
This paper illustrates the effects of stirring and graphene nanosheet (GNS) addition on the microstructure and mechanical behaviour of 393 hypereutectic $\mathrm{Al}-\mathrm{Si}$ alloys used in the diesel engine pistons. Two processing routes were applied to fabricate hypereutectic Al-Si alloys: The first route mainly depends on stirring $\mathrm{Al}-\mathrm{Si}$ alloys for 12 minutes at $400 \mathrm{rpm}$. The second one involves stirring Al-Si alloys for 2 minutes and then adding graphene nanosheets into the vortex, and consequently continue stirring for 10 minutes at $400 \mathrm{rpm}$. Results show that the distribution of the primary silicon was improved significantly in stir casting at both the edge and the center of the samples with relative reduction of $34 \%$ and $37 \%$ in the average particle size respectively. This average primary silicon size was further reduced by $17 \%$ with the addition of $1 \mathrm{wt} \%$ GNSs. GNS embedding into $\mathrm{Al}-\mathrm{Si}$ alloy matrix resulted in remarkable increase in hardness values of the nanocomposites compared to the cast alloy.
\end{abstract}

Keywords-aluminum; silicon; composite; hypereutectic; graphene

\section{INTRODUCTION}

The number of researches on the Al-Si alloys used in automotive industries has increased due to the demand of improving wear resistance and fatigue strength [1]. Conventional casting results in coarse and segregated microstructures, which lowers their mechanical properties. Therefore, improving the microstructural features can play an important role on the mechanical properties of these materials. Hypereutectic Al-Si alloys usually contain coarse primary silicon particles (PSPs), eutectic silicon and eutectic alpha phases. The presence of coarse primary silicon phase in these alloys has limited their industrial use [2]. Primary silicon has very hard particles, which significantly increase locally the wear resistance, however the brittleness of silicon and its cracking ease can easily expose the soft $\mathrm{Al}$ matrix to extreme wear [3-5]. Therefore, the appropriate solution is to fully refine the primary $\mathrm{Si}$, which can result in a considerable increment of the wear resistance for hypereutectic alloys [6,7]. Authors in [8] reported that specific casting techniques such as spray and centrifugal casting can refine the microstructure of the hypereutectic Al-Si alloys and uniform Si distribution. This refined microstructure and uniformed distribution can lead to improved strength, ductility and hardness.

On the other hand, further developments for the microstructure and consequently the mechanical properties can be achieved by reinforcing small particles into the Al matrix. Many researchers [9-13] pointed out that $\mathrm{Al}$ composites are the most promising materials to overcome the increasing demands of modern technology. Reinforcing of the Al matrix with nanoparticles or nanoplateletes has attracted significant attention due to their properties of augmented strength, elastic modulus, electrical and thermal conductivity. Some of these nanoscale materials are $\mathrm{SiC}, \mathrm{AL}_{2} \mathrm{O}_{3}, \mathrm{~B}_{4} \mathrm{C}, \mathrm{TiB}_{2}$ [14]. However, it is difficult to achieve a uniform distribution of the reinforcement material due to their poor wetability. Moreover, most of the reinforcement materials used to improve the properties of aluminum matrix composites, were hard ceramic particulates. These ceramic reinforcements have different density than that of aluminum, which increases the difficulty of proper mixing. The particles tend to sink or float when mixed with molten aluminum. Thus, it is extremely difficult to obtain a uniform distribution of ceramic particles, especially at nanoscale due to the increasing of surface area to volume ratio and surface energy, which enhances the formation of agglomerations [15]. Recently, researchers have introduced different types of nanoparticles in the production of $\mathrm{Al}$ composites, such as carbon nanotubes (CNTs) and graphene [9, 
16-21]. The incorporation of CNTs into the aluminum matrix resulted in an increasing in hardness, elastic modulus and indentation hardness as stated in. [17]. Authors in [19] reported that the graphene nanoplates embedding can potentially increase the strength and ductility of $\mathrm{Al}$ composites compared to monolithic aluminum. Authors in [16] showed that the addition of $0.5 \mathrm{wt} \%$ graphene nanoplates to aluminum increased strength and ductility over pure aluminum. Aggregation of CNTs and graphene nanoplates was the key challenge in these researches. A uniform distribution of such reinforcement was difficult to obtain. This can be attributed to some important factors such as the higher volume fraction, density, surface properties of the reinforcement, wetting purposes and processing parameters. As a result, graphene become an ideal reinforcement material for hypereutectic Al-Si alloys used in this work due to its unique two dimensional structure and outstanding physical and mechanical properties [22]. A combination of refining the primary $\mathrm{Si}$ phases and reinforcing the Al matrix with graphene nanosheets can lead to a proper microstructure and consequently enhanced alloy mechanical properties. Therefore, the present study aims to develop an in-house stir casting process for fabricating hypereutectic $\mathrm{Al}-\mathrm{Si} / \mathrm{Graphene}$ nanocomposites with refined and well distributed primary Si phases. The effects of cast stirring and graphene addition on morphology and hardness are investigated.

\section{EXPERIMENTAL METHODS}

\section{A. Materials}

The alloy used in the present work was 393 hypereutectic Al-Si alloy with addition of $\mathrm{Mg}$ powder and graphene nanosheets. The chemical composition of the base alloy is indicated in Table I. A-12 Graphene nanosheets were purchased from Graphene Laboratories Inc., USA with average thickness $<3 \mathrm{~nm}$ (between 3-8 graphene monolayers) and lateral dimensions $2-8 \mu \mathrm{m}$. Magnesium powder (230 mesh) was provided by Chemsupply-hu, Budapest, Hungary.

TABLE I. CHEMICAL PERCENTAGE OF 393 BASE ALLOY (WT\%)

\begin{tabular}{|c|c|c|c|c|c|c|}
\hline $\mathbf{S i}$ & $\mathbf{C u}$ & $\mathbf{M g}$ & $\mathbf{N i}$ & $\mathbf{F e}$ & $\mathbf{M n}$ & $\mathbf{A l}$ \\
\hline 22 & 0.2 & 0.4 & 2.1 & 0.6 & 0.0 & Bal \\
\hline
\end{tabular}

\section{B. Processing}

An in-house processing technique was used to cast $\mathrm{Al} /$ Graphene nanocomposites that can be used in automotive applications, especially in engine pistons. Graphene powder was dispersed in $100 \mathrm{ml}$ ethanol using CD-4810 ultrasonic for 30 minutes. The mixture was dried in oven at $80^{\circ} \mathrm{C}$ for $24 \mathrm{hrs}$, then quantity of $1 \mathrm{wt} \% \mathrm{Mg}$ was pre-mixed with 0.25 and $1 \mathrm{wt} \%$ graphene, separately in order to enhance the wettability, and therefore reduce nanosheet aggregations. The desired quantity of Al-Si alloy was melted in a resistance heating furnace at $750^{\circ} \mathrm{C}$ using a conical shaped graphite crucible and stirring was applied for 2 minutes at $400 \mathrm{rpm}$ [23]. The stirring action was started at low speed rate of $50 \mathrm{rpm}$ and increased slowly to 400 $\mathrm{rpm}$. The mixture was added directly into the vortex, and then stirring continued for another 10 minutes. The process was carried out in an argon gas environment to avoid oxidation. The slurry was poured in a hot cylindrical mould at uniform flow rate to avoid gas trapping, and then the mould was cooled at room temperature.

\section{Material Testing and Characterization}

Specimens were grinded, polished, etched with a $0.5 \% \mathrm{HF}$ queous solution and examined metallographically by an optical microscope. Microstructure and PSPs were measured and analyzed with Scentis image analyzer software (with 5\% error). Hardness testing of hypereutectic Al-Si/graphene nanocomposites was carried out on a Zwic-Roell ZHR tester. All samples were polished before conducting the test. A 100 kgf weight and 1/16" ball indenter were used in this study, at least five measurements were taken for each sample and the average value of the hardness was calculated.

\section{RESULTS AND DISCUSSION}

Primary silicon is the most important phase in hypereutectic Al-Si alloy microstructure. Pure silicon crystals structure in both primary and eutectic are the form that present in hypereutectic aluminum-silicon alloys. Silicon particle crystals are harder than any other phases, which can typically be found in aluminum casting alloys. Although the hypereutectic Al-Si alloy offers excellent wear resistance, lower density and thermal expansion coefficient, and superior dimensional stability at high temperature, the appearance of coarse primary $\mathrm{Si}$ crystals seriously impairs its strength, toughness and machinability, limiting its usage in industry [24]

\section{A. Microstructure}

The microstructures of hypereutectic Al-Si alloy for the ascast, stir casting and stir casting with various graphene additions at the sample edge and center are shown in Figures 1 (a), (b). The as-cast microstructure consists meanly of primary $\mathrm{Si}$, eutectic Si and eutectic $\alpha$. Figures 1(c), (d), (e) and (f) show that the stirring with or without the addition of graphene nanoplatelets can significantly affect the primary Si particle shape, size and distribution. It is well known that the use of mechanical stirring in semi-solid state can strongly promote refinement of the nondendritic structure due to the fragmentation of the primary $\mathrm{Si}$ crystals during semi-solid processing [25]. In current research work, the mechanical stirring resulted in a well refined microstructure. The primary Si particles in both edge and center of the samples tend to be finer and more globular. However, the primary Si particle distributions are still inhomogeneous especially in the center of the samples. The aggregation of the primary silicon particles can lead to unsatisfactory mechanical properties of stirred samples.

In addition to stirring, further primary $\mathrm{Si}$ particles refinements are obtained by the embedding of low volume fraction of graphene nanosheets into the Al matrix. It can be seen in Figures $1(\mathrm{~g})$ and $(\mathrm{h})$ that the addition of $1 \mathrm{wt} \%$ graphene nanoplatelets resulted in more finer and globular primary Si particles. Moreover, at the same volume fraction (1 $\mathrm{wt} \%$ ) a good primary Si particle distribution is obtained in both sample edge and center. This can be attributed to the presence of graphene nanosheets, which may hinder the growth of primary Si particles. 


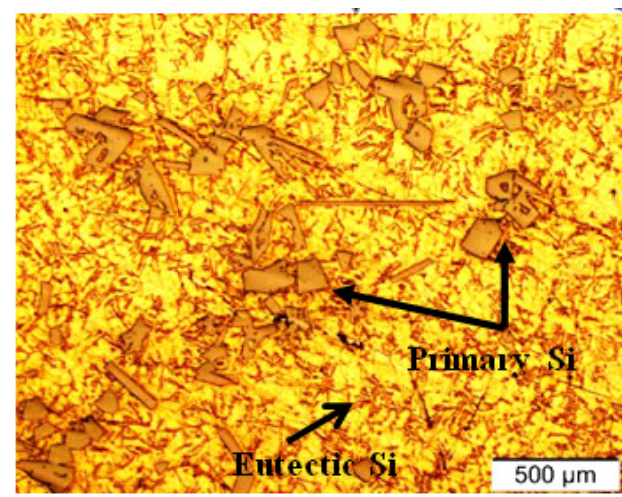

(a)

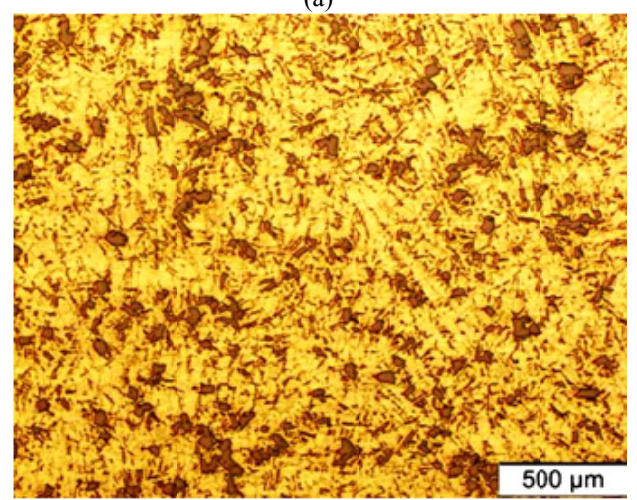

(c)

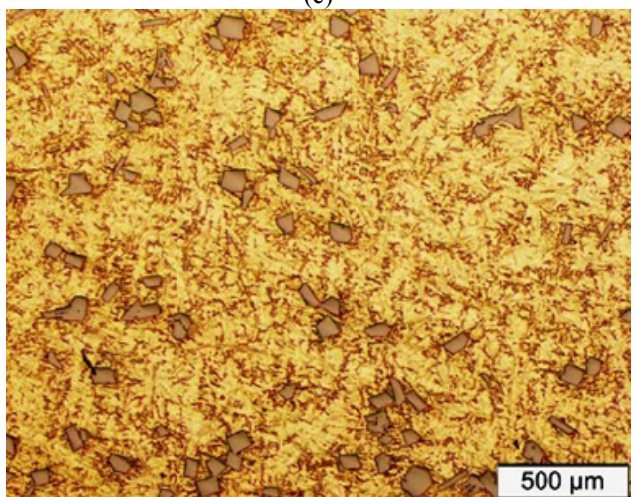

(e)

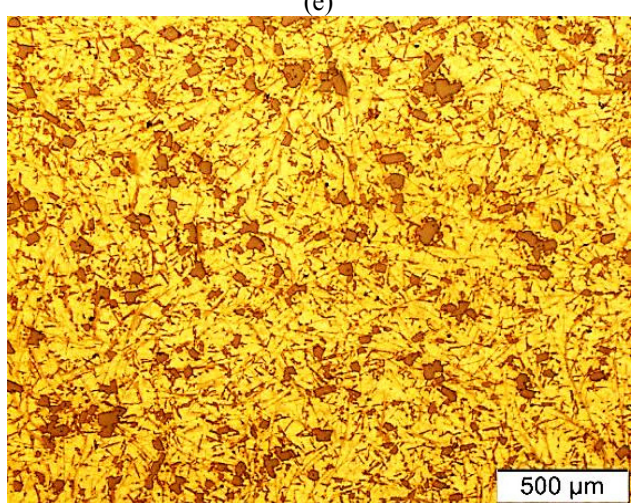

(g)

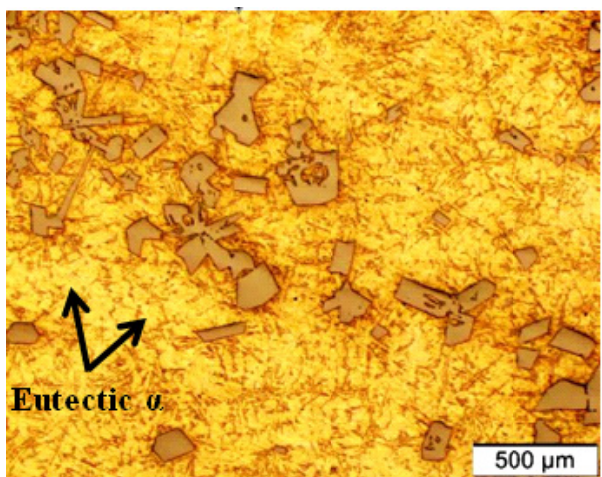

(b)

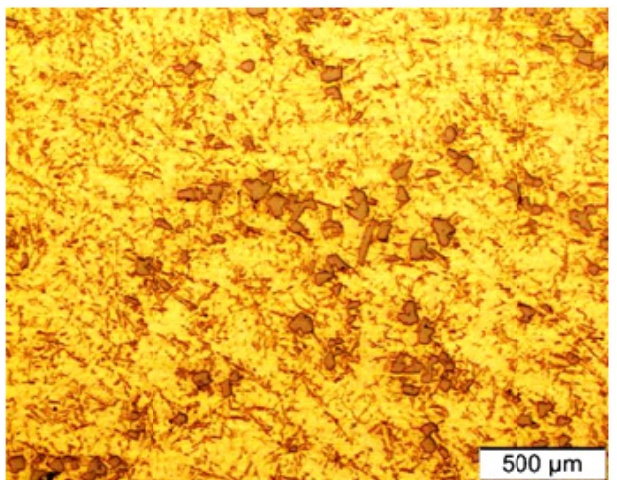

(d)

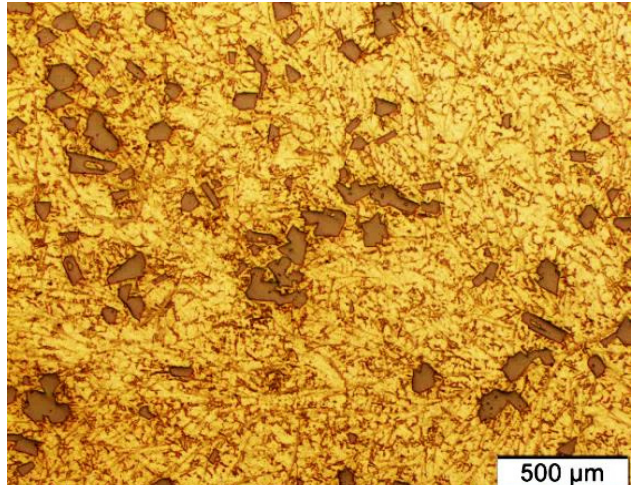

(f)

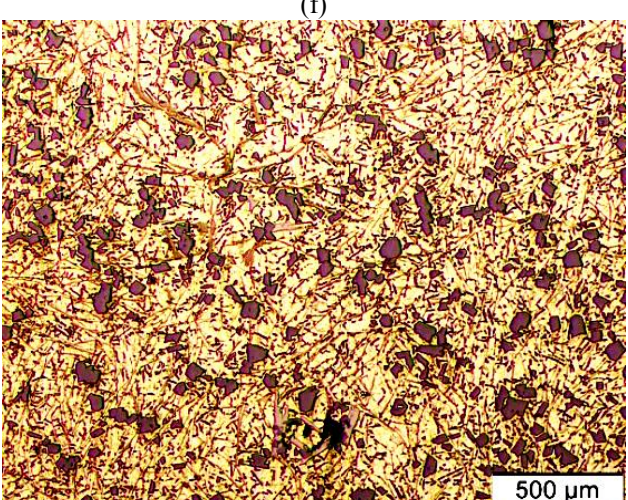

(h)

Fig. 1. Microstructure of hypereutectic Al-Si Alloy for as-cast, stir casting and stir casting with graphene additions at sample edge and center: a) and b) as-cast, c) and d) stir casting, e) and f) stir casting with $0.25 \mathrm{wt} \%$ graphene additions, g) and h) stir casting with $1 \mathrm{wt} \%$ graphene additions. 
Figure 2 shows the effect of stir casting and graphene additions on the primary Si particle size at both specimen's edge and center. It is clear that stirring and graphene addition of $1 \mathrm{wt} \%$ have a significant effect on the primary Si particle size. The unmodified as-cast samples microstructure contains primary Si particles with average particle size of $108 \mu \mathrm{m}$ and $97.5 \mu \mathrm{m}$ at the center and the edge respectively. Relatively, finer primary Si particles with average grain size of $68.75 \mu \mathrm{m}$ and $65 \mu \mathrm{m}$ at the center and the edge respectively are achieved by stir casting. Moreover, higher refinement of primary Si particles size of $51.5 \mu \mathrm{m}$ and $49.5 \mu \mathrm{m}$ are achieved by simultaneous stirring and addition of $1 \mathrm{wt} \%$ graphene nanosheets. Primary Si shape factor is increased by using stir casting and graphene addition, as shown in Figure 3. A primary Si with shape factor of 0.77 and 0.74 at the edge and the center of the sample is achieved by both stir casting and $1 \mathrm{wt} \%$ graphene nanoplatelets addition. Authors in [26] stated that, in a hypereutectic $\mathrm{Al}-20 \mathrm{wt} \% \mathrm{Si}$ alloy, the morphology of the primary Si particles (PSPs) mostly has a shape factor of 0.85 , with a topology of either a regular octahedral shape or a twinned shape, and because of the influence of the surrounding microstructure, the morphology of the PSPs would normally exhibit a combined morphology, i.e. different from the perfect octahedral shape or twinned shape. Morphology of the primary Si particles was analyzed by employing the synchrotron X-ray tomography technique and a growth transition model was developed. It was found that both octahedral and/or twinned shapes were a transition from a facetted growth unit, which had the half octahedron morphology and evolved from a spherical nucleation. After octahedral and/or twinned shapes and with further growth of the silicon particles, the formations of hollow hoppers due to the mismatch of the growth velocity along different directions were obtained.

PSPs with irregular shape that probably contains a combined morphology of octahedral and twinned shapes in ascast structure of the current research work indicated that the growth of PSPs reached its final state as seen in Figure 4(a). Stir casting with or without graphene additions can probably change the degree of under cooling during the growth of PSPs just after nucleation. With relatively larger under cooling, the magnitude of the Jackson factor at different crystallographic planes is lower than the critical value for facetted growth, which resulted in grew of a non-facetted mode. Whereas, by decreasing the under cooling, Jackson factor approaches its critical value and the facetted growth mode is performed [27, 28]. Figure 4 shows the effect of stirring and graphene nanosheets addition on the primary Si particles morphology of 393 hypereutectic-Si alloy and hollow hoppers formation. The relationship between PSPs morphology and stirring with or without graphene nanoplatelets additions can be observed from the values of shape factor shown in Figure 3 and the microstructure shown in Figure 4. PSPs morphology of facetted growth as in as-cast microstructure changed to a combination of facetted growth and octahedral /twinned shapes by using stir casting. However, the addition of $1 \mathrm{wt} \%$ graphene nanosheets combined with stirring resulted in a structure with mainly octahedral /twinned shapes as seen in Figure 4(d). The hollow hoppers that usually formed in PSPs in as-cast structure are decreased with stir casting process and graphene nanosheets addition. A few numbers of hollow hoppers that formed in stir casting combined with graphene additions appeared in a black color as indicated by the arrows. This can be probably caused by some graphene nanoplatelets agglomerations.

\section{B. Hardness}

The effects of stir cast and stir cast with graphene additions of 393 hypereutectic Al-Si alloys on the hardness are presented in Figure 5. It was found that hardness is increased with stir casting processing due to the PSPs refining. Maximum hardness values of $73.8-75 \mathrm{HRB}$ can be achieved by the addition of graphene nanosheets during stir casting. This can be attributed to the presence of fine PSPs and their uniform distribution.

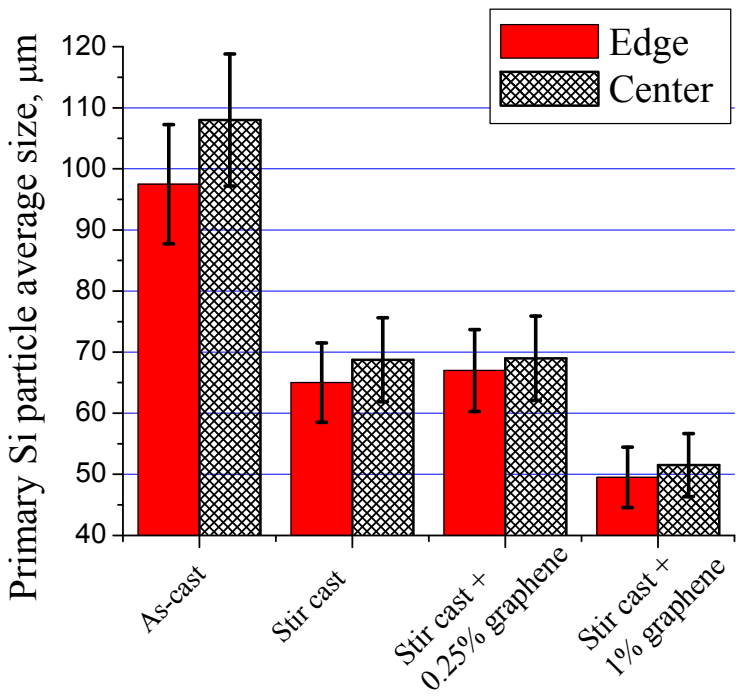

Fig. 2. Primary Si particle size as a function of stir casting and graphene additions.

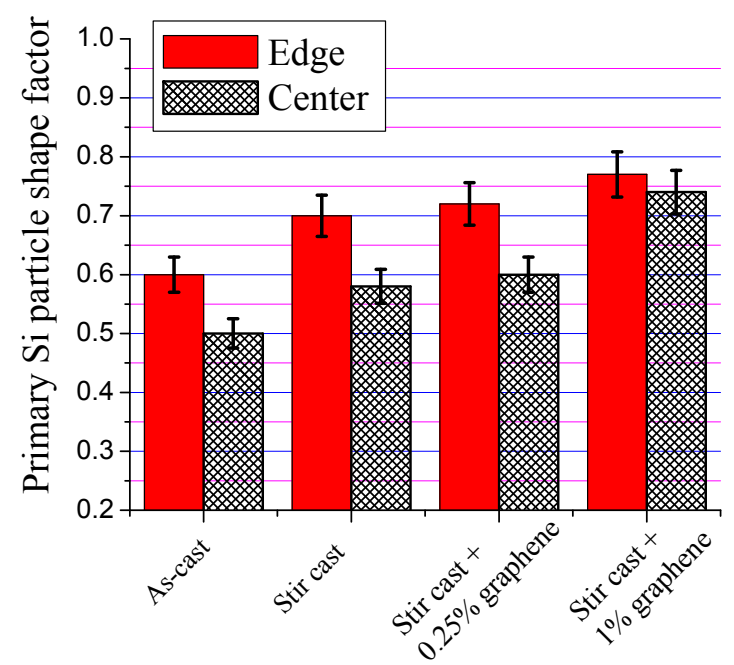

Fig. 3. Primary Si shape factor as a function of stir casting and graphene nanosheets additions. 


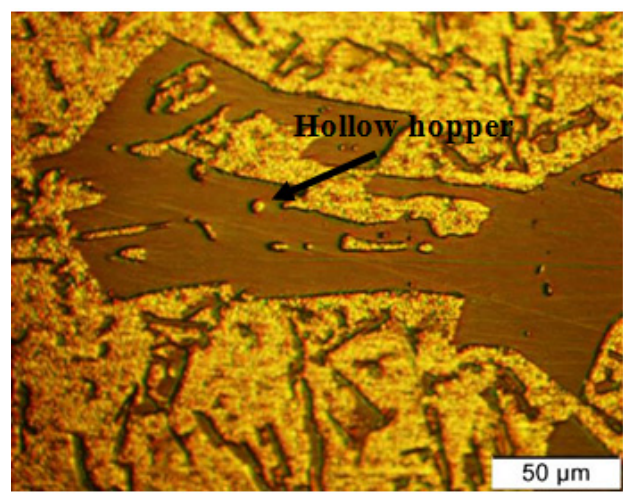

(a)

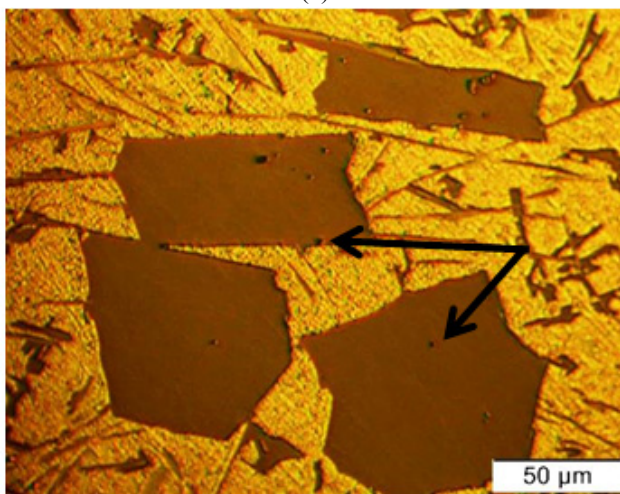

(c)

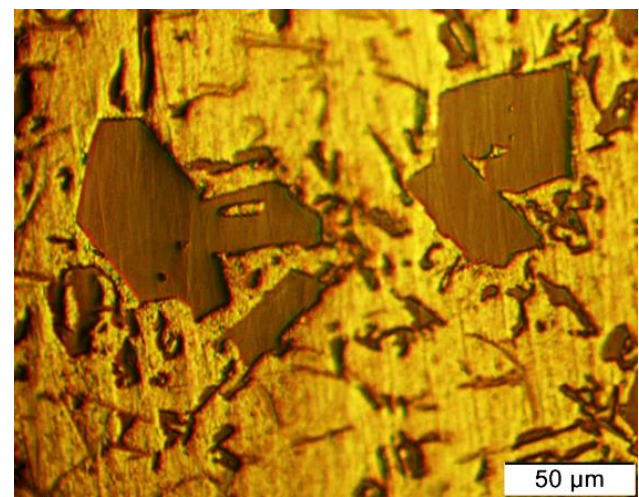

(b)

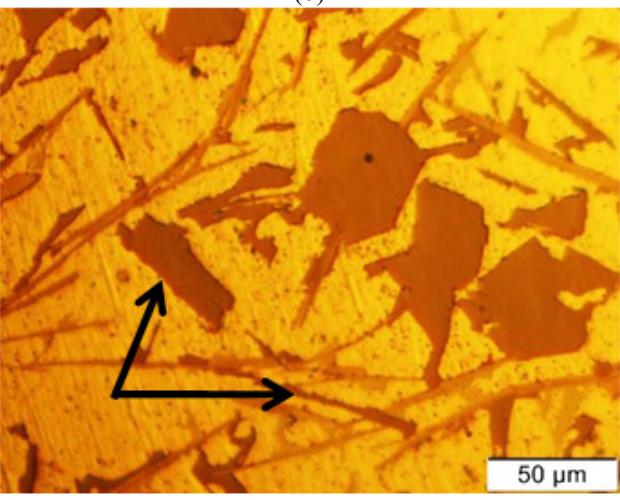

(d)

Fig. 4. Efects of stirring and graphene addition on PSPs morphology of 393 hypereutectic Al-Si alloy: a) as-cast, b) stir cast, c) stir cast with $0.25 \mathrm{wt} \%$ graphene nanosheets, d) stir cast with $1 \mathrm{wt} \%$ graphene nanosheets, arrow denoteblack hollow hoppersinside primary Si particles and black points at thir boundaries.

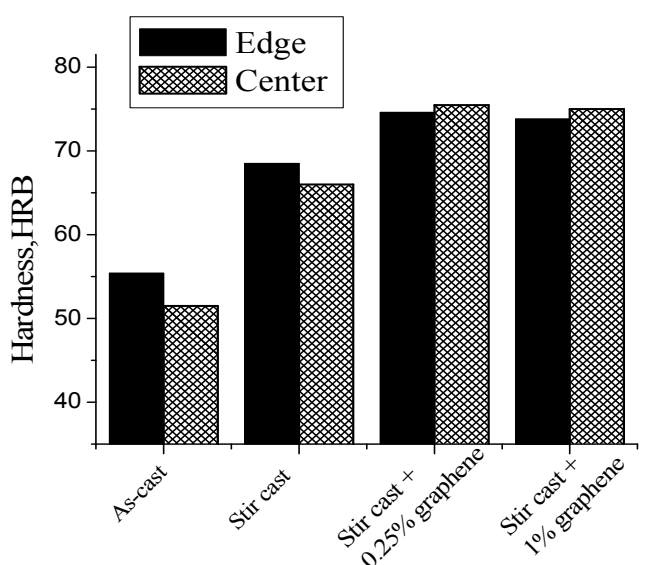

Fig. 5. Hardness measurements of as-cast, stir cast and stir cast with graphene additions of 393 hypereutectic Al-Si alloy.

\section{CONCLUSION}

For a hypereutectic Al-Si alloy, a combination of stir casting and graphene nanosheets addition had a significant influence on the PSPs average particle size and distribution. This particle size refining and improved distribution, especially in the center of the sample can be related to their working as nucleating agent and/or hinders for the PSPs growth.
Moreover, hardness values can increase remarkably with simultaneous application of stirring and addition of low volume fraction of graphene nanosheets. Future investigations are needed to clearly define the role of graphene and its effects on the PSPs and eutectic structure in the 393 hypereutectic Al-Si alloy.

\section{ACKNOWLEDGMENT}

Acknowledgments to the Deanship of Scientific Research, University of Hail, Saudi Arabia, for funding and supporting this project (reference number: BA-1505).

\section{REFERENCES}

[1] H. Ye, "An overview of the development of Al-Si alloy based material for engine applications", Journal of Materials Engineering and Performance, Vol. 12, No. 3, pp. 288-297, 2003

[2] F. C. Robles Hernandez, J. H. Sokolowski, "Thermal analysis and Microscopical characterization of Al-Si hypereutectic alloys", Journal of Alloys and Compounds, Vol. 419, No. 1-2, pp. 180-190, 2006

[3] J. H. Sokolowski, J. Mazurek, "The Structure and Matrix Microhardness of the 319 Aluminum Alloy After Isothermal Holding During the Solidification Process", AFS Trans, Vol. 95, pp. 373-376, 1987

[4] L. Lasa, J. M. Rodriguez-Ibabe, "Wear behaviour of eutectic and hypereutectic $\mathrm{Al}-\mathrm{Si}-\mathrm{Cu}-\mathrm{Mg}$ casting alloys tested against a composite brake pad", Materials Science and Engineering: A, Vol. 363, No. 1-2, pp. 193-202, 2003 
[5] T. Hejwowski, A. Weronski, "The Effect of Thermal Barrier Coatings on Diesel Engine Performance",Vacuum, Vol. 65, No. 3-4, pp. 427-432, 2002

[6] F. C. Robles Hernandez, Improvement in functional characteristics of aluminum-silicon cast components through the utilization of a novel electromagnetic treatment of liquid melts, PhD Thesis, University of Windsor, 2004

[7] F. C. Robles Hernandez, J. H. Sokolowski, M. Kasprzak, "Electromagnetic Refinement of the 319 Aluminum Structure", 43rd Conference of Metallurgists, 2004

[8] K. Raju, A. P. Harsha, S. N. Ojha, "Effect of processing techniques on the mechanical and wear properties of Al-20Si alloy", Transactions of the Indian Institute of Metals, Vol. 64, No. 1, pp. 1-5, 2011

[9] S. N. Alam, L. Kumar, "Mechanical Properties of Aluminium Based Metal Matrix Composites Reinforced with Graphite Nanoplatelets", Materials Science and Engineering: A, Vol. 667, pp. 16-32, 2016

[10] A. K. Geim, K. S. Novoselov, "The Rise of Graphene", Nature Materials, Vol. 6, pp. 183-191, 2007

[11] A. C. Ferrari, J. C. Meyer, V. Scardaci, C. Casiraghi, M. Lazzeri, F. Mauri, S. Piscanec, D. Jiang, K. S. Novoselov, S. Roth, A. K. Geim, "Raman Spectrum of Graphene and Graphene Layers", Physical Review Letters, Vol. 97, No. 18, p. 187401, 2006

[12] A. H. C. Neto, F. Guinea, N. M. R. Peres, K. S. Novoselov, A. K. Geim, "The electronic properties of graphene", Reviews of Modern Physics, Vol. 81, No. 1, pp. 109-162, 2009

[13] L. M. Viculis, J. J. Mack, O. M. Mayer, H. T. Hahn, R. B. Kaner, "Intercalation and exfoliation routes to graphite nanoplatelets", Journal of Materials Chemistry, Vol. 15, No. 9, pp. 974-9778, 2005

[14] S. Johny James, K. Venkatesan, P. Kuppan, R. Ramanujam, "Hybrid Aluminium Metal Matrix Composite Reinforced with SiC and TiB2", Procedia Engineering, Vol. 97, pp. 1018-1026, 2014

[15] A. Mazahery, M. Ostadshabani, "Investigation on mechanical properties of nanoAl2O3 reinforced aluminum matrix composites", Journal of Composite Materials, Vol.45, No. 24, pp.2579-2586, 2011

[16] J. L. Li, Y. C. Xiong, X. D. Wang, S. J. Yan, C Yang, W. W. He, J .Z. Chen, S. Q. Wang, X. Y. Zhang, S. L., Dai, "Microstructure and Tensile Properties of Bulk Nanostructured Aluminum/Graphene Composites Prepared via Cryomilling”, Materials Science and Engineering: A, Vol. 626, pp. 400-405, 2015

[17] M. Raviathul Basariya, V. C. Srivastava, N. K. Mukhopadhyay, "Microstructural characteristics and mechanical properties of carbon nanotube reinforced aluminum alloy composites produced by ball milling", Materials and design, Vol. 64, pp. 542-549, 2014

[18] W. J. Kim, Y. J. Yu, "The effect of the addition of multiwalled carbon nanotubes on the uniform distribution of TiC nanoparticles in aluminum nanocomposites”, Scripta Materialia, Vol. 72-73, pp. 25-28, 2014

[19] S. J. Yan, S. L. Dai, X. Y. Zhang, C. Yang, Q. H. Hong, J. Z. Chen, Z. M. Lin, "Investigating aluminum alloy reinforced by graphene nanoflakes", Materials Science and Engineering: A, Vol. 612, pp.440444, 2014

[20] J. Wang, Z. Li, G. Fan, P. Huanhuan, C. Zhixin, D. Zhang, "Reinforcement with graphene nanosheets in aluminum matrix composites", Scripta Materialia, Vol.66, pp. 594-597, 2012

[21] W. Yang, Q. Zhao, L. Xin, J. Qiao, J. Zou, P. Shao, Z. Yu, Q. Zhang, G. $\mathrm{Wu}$, "Microstructure and mechanical properties of graphene nanoplates reinforced pure $\mathrm{Al}$ matrix composites prepared by pressure infiltration method", Journal of Alloys and Compounds, Vol.732, pp.748-758, 2018

[22] Z. Hu, G. Tong, D. Lin, C. Chen, H. Guo, J. Xu, L. Zhou, "Graphenereinforced metal matrix nanocomposites - a review", Materials Science and Technology, Vol. 32, No. 9, pp. 930-953, 2016

[23] R. G. Bhandare, P. M. Sonawane, "Preparation of Aluminium Matrix Composite by Using Stir Casting Method", International Journal of Engineering and Advanced Technology, Vol.3, No.2, pp. 61-65, 2013

[24] R. Wang, L. Weihua, "Hypereutectic Al-Si Alloy with Completely Nodular Eutectic Silicon: Microstructure and Process", International Journal of Materials Science and Applications, Vol. 5, No. 6, pp. 277283, 2016

[25] H. Takagi, Y. Uetani, A. Dohi, T. Yamashita, K. Matsuda, S. Ikeno, "Effects of Mechanical Stirring and Vibration on the Microstructure of Hypereutectic Al-Si-Cu-Mg Alloy Billets", Materials Transactions, Vol. 48, No. 5, pp. 960-966, 2007

[26] J. Wang, Z. Guoa, J. L. Song, W. X. Hu, J. C. Li, S. M. Xiong, "On the growth mechanism of the primary silicon particle in a hypereutectic Al$20 \mathrm{wt} \% \mathrm{Si}$ alloy using synchrotron X-ray tomography", Materials and Design, Vol. 137, pp. 176-183, 2018

[27] X. Yang, K. Fujiwara, R. Gotoh, K. Maeda, J. Nozawa, H. Koizumi, S. Uda, "Crystal and faceted dendrite growth of silicon near (100)", Acta Materia, Vol. 60, No. 8, pp.3259-3267, 2012

[28] W. Wang, X. F. Bian, J. Qin, S. I. Syliusarenko, "The atomic-structure changes in Al-16 pct Si alloy above the liquidus", Metallurgical and Materials Transactions A, Vol. 31, No. 9, pp. 2163-2168, 2000 